\title{
Cobertura vacinal e mortalidade infantil em Campina Grande, PB, Brasil
}

\author{
Vaccination coverage and child mortality in Campina Grande, PB, Brazil \\ Cobertura vacunal y mortalidad infantil en Campina Grande, PB, Brasil
}
Inacia Sátiro Xavier de França', Daniela da Nóbrega Simplício', Fabiana Paulino Alves ${ }^{1}$, Virgínia Rossana de Sousa Brito'

'Universidade Estadual da Paraíba. Departamento de Enfermagem. Campina Grande, PB

Submissão: $09 / 04 / 2008$

Aprovação: $26 / 02 / 2009$

\section{RESUMO}

Estudo transversal para investigar cobertura vacinal e mortalidade de crianças menores de um ano de idade, identificar recursos, ações e dificuldades para alcançar meta vacinal. Participaram 2.206 crianças e 62 enfermeiros das Unidades Básicas de Saúde da Família. Utilizou-se formulário e entrevista semi-estruturada. Identificaram-se ações direcionadas à saúde da criança; dispersão entre o número de crianças cadastradas nas UBSF e o de nascidos vivos; oscilações dos percentuais de vacinação, ora aproximando-se da meta, ora afastando-se. A resistência dos pais a vacinação, falta de insumos e de ACS em algumas UBSF foram as principais barreiras enfrentadas para atingir a meta. O índice de mortalidade infantil ficou abaixo de 20, confirmando o impacto da cobertura vacinal.

Descritores: Programas de imunização; Imunização em massa, vacinação, mortalidade infantil.

\begin{abstract}
Cohort study to investigate mortality and immunization coverage of children under one year of age, identify resources, actions and difficulties to achieve goal vaccine. 2,206 children and 62 nurses from Family Health Basic Unities participated in the study. A questionnaire and semi-structured interview were used. Children's health targed acctions, the dispersion of the registered children in this unities and the number of live births; the oscillations of the percentage of vaccination, now closer or distant of the goal. The resistance of parent's vaccination, lack of inputs and ACS in some UBSF were the main barrier faced to achieve the goal. The rate of infant mortality was below 20, confirming the impact of immunization coverage.

Descriptors: Immunization programs; Mass immunization; Vaccination; Infant Mortality

\section{RESUMEN}

Estudio transversal para investigar cubierta vacunal y mortalidad de niños menores de un año de edad, identificar recursos, acciones y dificultades para alcanzar meta vacunal. Participaron 2.206 niños y 62 enfermeros de las Unidades Basicas de Salud de la Familia. Se utilizó formulario y entrevista semi estructurada. Se identificaron acciones orientadas a la salud del niño; dispersión entre el número de niños registrados en las unidades y los de nacidos vivos; oscilaciones de los porcentajes de vacunación, ora aproximándose de la meta, ora alejándose. La resistencia de los padres a vacunación, falta de insumos y de ACS en algunas unidades fueron las principales barreras enfrentadas para atingir la meta. El índice de mortalidad infantil se euedo abajo de 20, confirmando el impacto de la cubierta vacunal. Descriptores: Programas de inmunización; Inmunización masiva; Vacunación; Mortalidad infantil.
\end{abstract}




\section{INTRODUÇÃO}

As primeiras campanhas de vacinação em solo brasileiro datam de 1804. A partir de então, o país vem desenvolvendo estratégias diversas como campanhas, coberturas, varreduras, rotinas Que determinaram, em 1942, a eliminação da febre amarela urbana; em 1973, da varíola e, em 1989, da poliomielite. Atualmente, mantém sob controle o sarampo, o tétano neonatal, as formas graves de tuberculose, a difteria, o tétano acidental e a coqueluche ${ }^{(1)}$.

Em 18 de setembro de 1973, o governo brasileiro criou o Programa Nacional de Imunização-PNI, uma estratégia de prevenção e/ou controle da incidência de doenças infectocontagiosas. Esse programa tem como meta vacinar todos os brasileiros em todas as fases da sua vida. O impacto desse programa é identificado através da cobertura vacinal, um indicador de saúde acerca do percentual de crianças menores de um ano de idade imunizadas segundo o tipo de vacina, em determinada localidade e no ano. O percentual relativo a cobertura vacinal serve para estimar o nível de proteção da população infantil contra doenças selecionadas, evitáveis por imunização, devido o cumprimento do esquema básico de vacinação ${ }^{(2)}$.

Ao longo de seus 30 anos, o PNI conseguiu eliminar, ou manter sob controle, as doenças imunopreveníveis. Entretanto, o alcance das metas vacinais é dificultado pela inacessibilidade dos vacinadores às residências e pela crença popular de Que a vacinação de rotina não requer atenção ${ }^{(I)}$. E percebe-se Que o medo das possíveis reações da vacina é um outro fator negativo para o alcance da cobertura vacinal. Consideradas essas barreiras físicas e atitudinais há Que se perguntar: Estamos vacinando todas as crianças na faixa etária de zero a 01 ano? Se não estamos, Quais as dificuldades para alcançar essa meta?

A possibilidade de Que nem todas as pessoas estão imunizadas faz com Que o PNI desenvolva estratégias de cobertura objetivando vacinar $100 \%$ das crianças menores de um ano com todas as vacinas elencadas no calendário básico. Para alcançar essa meta, ocorre pactuação entre os conselhos municipais, estaduais e nacional de saúde e utiliza-se de estratégias de promoção da saúde para possibilitar à sociedade maior compreensão sobre os benefícios das vacinas para a saúde individual e coletiva.

No Brasil, de acordo com a Portaria No 597/2004 (3) , as vacinas recomendadas para crianças menores de um ano são as seguintes: BCG - ID (dose única), vacina contra hepatite B (3 doses), vacina oral contra pólio-VOP (3 doses), vacina tetravalente-DTP + Hib (3 doses), vacina contra febre amarela (dose única) e vacina SRCtríplice viral: sarampo, rubéola e cachumba (dose única).

Cumprir o calendário de vacinação infantil também é uma obrigação instituída pelo Estatuto da Criança e do Adolescente Que em seu Artigo $7^{\circ}$ assegura à esses indivíduos o direito a proteção á vida e à saúde, através da efetivação de políticas sociais públicas Que permitam o nascimento e o desenvolvimento sadio e harmonioso, em condições dignas de existência ${ }^{(4)}$.

Essa expectativa motiva a realização de estudos Que retratem se as ações de imunoprevenção estão gerando impacto compatível com a meta estabelecida pelo PNI de alcançar 100\% das crianças com menos de um ano de vida e se está ocorrendo a redução da morbi-mortalidade infantil.

Dados do IBGE estabelecem Que até o ano 2000 o CMI em
Campina Grande-PB era de 47,76 por mil e a esperança de vida ao nascer era de 63,47 anos $^{(5)}$. Esse índice se contrapõe ao CMI considerado aceitável pela Organização Mundial da Saúde (OMS) Que é de 10 mortes para cada mil nascimentos ${ }^{(2)}$.

Ao considerar esses dados, elaboramos as seguintes indagações: Em Campina Grande-PB, as doenças imunopreveníveis em crianças de zero a 01 ano de idade estão sob controle? Se estão, de Que morrem as crianças nessa faixa etária? Se a vacinação das crianças situadas nessa faixa etária não alcança a meta do PNI, Quais são as dificuldades enfrentadas pelos profissionais de saúde para o alcance dessa meta? A busca de respostas para esses Questionamentos motivou esse estudo Que tem como objeto a cobertura vacinal de crianças de zero a um ano.

Objetivou-se investigar o número de crianças cadastradas nas UBS de Campina Grande-PB no período de 2000 a 2005; se nesse período o município atingiu a cobertura vacinal das crianças menores de um ano de vida. Quais as causas de óbitos em crianças menores de um ano de idade nesse município. Identificar os recursos disponíveis para o planejamento das ações de imunização infantil. E Quais as possíveis barreiras enfrentadas pelos vacinadores para alcançar a meta 100\% de vacinação infantil proposta pelo PNI.

\section{METODOLOGIA}

Trata-se de um estudo transversal realizado na cidade de Campina Grande-PB, no período de agosto de 2006 a julho de 2007.

A cidade de Campina Grande-PB dista 136 Km da capital João Pessoa e situa-se na zona Centro-Oriental da Paraíba, no planalto da Borborema, no trecho mais alto de suas escarpas, sendo considerada uma das cidades mais importante do interior do norte/ nordeste brasileiro. Polariza um universo de cinco microrregiões homogêneas, num total de $23.960 \mathrm{~km}^{2}$ Que correspondem a $43 \%$ do território paraibano e $40 \%$ da população do Estado, constituindo o "Compartimento da Borborema". A região metropolitana campinense conta com uma população de 379. 87I habitantes, densidade demográfica de $612 \mathrm{hab} / \mathrm{km}^{2}$ e atualmente, o município conta com 55 UBSF, atendendo a um total de 112.686 famílias cadastradas $^{(6)}$.

A pesquisa foi realizada junto a Secretaria Municipal de Saúde de Campina Grande e das Unidades Básicas de Saúde da Família(UBSF) situadas na zona urbana desse município.

A população foi composta pelo total de 2.206 crianças cadastradas no Programa Saúde da Criança-PSC no período 2000 a 2005, e por 75 enfermeiros atuantes nas UBSF dessa cidade.

Selecionaram-se duas amostras: A amostra 01, do tipo não probabilística, composta pelas 2.206 crianças menores de um ano inscritas nos sistemas de informação da Secretaria Municipal de Saúde. E a amostra 02, composta por 62 enfermeiros atuantes nas UBS Que aceitaram participar do estudo.

Para a amostra 01 obtiveram-se dados secundários nos registros relativos aos seguintes Sistemas de Informações: Sistema de Informação sobre Mortalidade(SIM), Sistema de Informação sobre Nascidos Vivos(SINASV) e Sistema de Informação da Atenção Básica(SIAB). Utilizou-se um formulário com Questões objetivas acerca das variáveis: número de crianças nascidas vivas e residentes na zona urbana de Campina Grande-PB, número de crianças 
menores de um ano com esquema de vacinação completo, e o número e a causa de óbitos de crianças menores de um ano, de ambos os sexos, no período selecionado para o estudo. Para calcular o CMI utilizou-se o método direto, preconizado pelo Ministério da Saúde que é da seguinte ordem:

$$
\mathrm{CMI}=\frac{\mathrm{N}^{\mathrm{o}} \text { de óbitos de residentes }<01 \text { ano }}{\mathrm{N}^{\mathrm{o}} \text { de nascidos vivos de mães residentes }} \times 1.000
$$

Os dados da amostra 02 foram coletados através de entrevista semi-estruturada com os enfermeiros atuantes nas UBSF, contendo Questões acerca das variáveis: recursos disponíveis para o planejamento das ações de imunização infantil, ações desenvolvidas pela equipe do PSC para reduzir a morbi-mortalidade infantil e possíveis barreiras enfrentadas pelos vacinadores para alcançar a meta $100 \%$ de vacinação infantil proposta pelo PNI.

Quando da análise, utilizou-se a estatística descritiva, calculandose as taxas de vacinação por doença imunoprevenível e as taxas de morbidade e mortalidade infantil, global e específica, atribuindo-se a causa mortis. Os dados relativos às entrevistas com os enfermeiros foram agrupados e Quantificados por semelhança das respostas e, em seguida, calculados os percentuais de cada agrupamento em consonância com o número total de respondentes e o tamanho da amostra. Os resultados foram distribuídos em gráficos, tabelas e Quadros.

Após a autorização da Secretaria Municipal de Saúde de Campina Grande-PB para a realização do estudo, o projeto foi aprovado pelo Comitê de Ética em Pesquisa da Universidade Estadual da Paraíba (UEPB), sob o no 01.06.0.133.000-07. Durante a investigação, os pesquisadores obtiveram o consentimento livre e esclarecido dos participantes conforme determina a Resolução № 196/96 do Conselho Nacional de Saúde para as pesquisas envolvendo seres humanos.

\section{RESULTADOS E DISCUSSÃO}

As UBSF constituem um modelo de assistência destinado a cuidar da saúde da população que lhe é adscrita. Cada UBSF pode atuar com uma ou mais equipes do Programa Saúde da Família(PSF), conforme o número de famílias de sua área de atuação. Cada equipe PSF prioriza ações de prevenção, promoção e recuperação da saúde das pessoas, de forma integral e contínua ${ }^{(7)}$. No caso específico das ações destinadas a saúde da criança, é imprescindível o conhecimento da relação nascidos vivos-cadastro anual das crianças nas UBSF.

A Tabela I apresenta a disparidade entre o número anual de nascidos vivos e o de crianças cadastradas nas UBSF. Dentre as explicações possíveis para esse fenômeno destacam-se: A cidade atua como "Pólo de Referência na Assistência a Saúde" para cidades circunvizinhas e até mesmo de outros Estados. Os registros de óbito e de nascimento são feitos no e pelo local de ocorrência do evento. Dessa forma, o dado que mais interessa ao setor saúde é o relacionado ao local de residência, mas existem os casos em Que a gestante, principalmente aQuela de alto risco, vem para o município pela assistência, ficando internada por várias semanas e, após o parto, algumas crianças ficam internas por dias ou meses, são registradas e vão para o local de origem da família; normalmente Quando atingem o peso necessário para iniciar vacinação.

Outros motivos para a disparidade entre os dados do Quadro I é o fato do município contar com 55 equipes de saúde e, portanto, a cobertura populacional é menor Que $75 \%$.Assim, existe no município microáreas descobertas, com maior concentração de crianças e, conseqüentemente, sem acompanhamento e informações, Além desses motivos, pode ter diminuído o números de nascimentos na área adscrita às UBSF, o êxodo das famílias ou a subnotificação.

Na Tabela 2, devido o cálculo do índice de cobertura vacinal se basear no número de doses aplicadas, sob a influência da cobertura dos nascimentos de ocorrência, observa-se Que em relação às vacinas BCG, anti Pólio e SRC os índices extrapolaram 100\% de cobertura. Também identificou-se Que a meta proposta pelo Programa Nacional de Imunização de, no mínimo, 95\% de cobertura vacinal na faixa etária de 0 a 1 ano de idade, não está sendo, plenamente, atingida.

Em relação ao resultado "Dado ausente" apresentado no Quadro 2, convém esclarecer Que, em se tratando da vacina tetravalente (DTP+HIB), essa foi introduzida no calendário nacional em 2002, por isso não tem registro anterior. Antes de sua introdução no calendário vacinal administrava-se e registrava-se, separadamente, DTP e HIB. Por isso, nesse estudo, considerou-se os índices de DTP e HIB administradas no período 2000 a 2002. Convém considerar Que, em 2002, o índice 50,37 para a Tetravalente pode ser conseQüência da organização do serviço de imunização em nível nacional.

Os índices diminutos para a cobertura vacinal contra a febre amarela são justificáveis porque a Portaria no 597 só a incluiu no calendário de vacinação no ano 2004. Outra justificativa possível para a insipiência desse dado é o entendimento do senso comum

Tabela 1. Relação numérica entre nascidos vivos e crianças cadastradas nas UBSF da zona urbana de Campina Grande-

\begin{tabular}{|c|c|c|c|c|c|c|}
\hline \multirow{2}{*}{ Ano } & \multicolumn{3}{|c|}{ Nascidos vivos } & \multicolumn{3}{|c|}{ Crianças < de I ano cadastradas na atenção básica } \\
\hline & $\mathrm{M}$ & $\mathrm{F}$ & Total & $\mathrm{M}$ & $\mathrm{F}$ & Total \\
\hline 2000 & 3.448 & 3.273 & 6.721 & 58 & 68 & 126 \\
\hline 2001 & 3.379 & 3.387 & 6.766 & 33 & 27 & 60 \\
\hline 2002 & 3.513 & 3.380 & 6.893 & 700 & 736 & 1.436 \\
\hline 2003 & 3.195 & 3.219 & 6.414 & 136 & 145 & 281 \\
\hline 2004 & 3.207 & 3.048 & 6.255 & 87 & 101 & 188 \\
\hline 2005 & 3.155 & 3.097 & 6.252 & 53 & 62 & 115 \\
\hline Total & 19.897 & 19.404 & 39.301 & 1.067 & 1.139 & 2.206 \\
\hline
\end{tabular}
PB, no período de 2000 a 2005. 
Tabela 2. Cobertura da imunização e imunobiológicos administrados a crianças < de 1 ano na zona urbana de Campina Grande-PB no período de 2000 a 2005.

\begin{tabular}{lcccccc}
\hline \multirow{2}{*}{ Vacina administrada } & \multicolumn{5}{c}{ Percentual de cobertura anual } \\
\cline { 2 - 6 } & $\mathbf{2 0 0 0}$ & $\mathbf{2 0 0 1}$ & $\mathbf{2 0 0 2}$ & $\mathbf{2 0 0 3}$ & $\mathbf{2 0 0 4}$ & $\mathbf{2 0 0 5}$ \\
\hline BCG & 114 & 140,52 & 148,12 & 177,53 & 220,80 & 214,38 \\
Hepatite B & 92,68 & 89,15 & 91,08 & 95,01 & 86,12 & 93,72 \\
Tetravalente-DTP+Hib & Dado ausente & Dado ausente & 50,37 & 96,35 & 90,26 & 95,14 \\
DTP & 85,79 & 96,13 & $41,76 \%$ & - & - & - \\
HIB & 90,91 & 79,35 & 40,83 & - & - & - \\
Anti poliomielite & 87,50 & 95,71 & 104,34 & 95,79 & 86,93 & 96,63 \\
Vacina SRC (tríplice viral) & 46,30 & 99,83 & 98,30 & 112,20 & 110,74 & 99,56 \\
Anti febre amarela & 0,04 & Dado ausente & 0,02 & Dado ausente & 0,04 & 0,04 \\
\hline
\end{tabular}

Tabela 3. Número de óbitos e causa mortis de crianças de zero a um ano na cidade de Campina Grande-PB no período de 2000 a 2005.

\begin{tabular}{|c|c|c|c|c|c|c|c|c|c|c|c|c|c|}
\hline \multirow{2}{*}{ Causa mortis de crianças $<0$ I ano } & \multicolumn{2}{|c|}{2000} & \multicolumn{2}{|c|}{2001} & \multicolumn{2}{|c|}{2002} & \multicolumn{2}{|c|}{2003} & \multicolumn{2}{|c|}{2004} & \multicolumn{2}{|c|}{2005} & \multirow{2}{*}{ Total } \\
\hline & $M$ & $\mathrm{~F}$ & $M$ & $\mathrm{~F}$ & $M$ & $F$ & $M$ & $F$ & $\mathrm{M}$ & $\mathrm{F}$ & $M$ & $F$ & \\
\hline Doença infecciosa ou parasitária & 9 & 6 & 3 & - & 3 & 1 & 2 & 3 & 4 & 3 & 3 & 3 & 40 \\
\hline Neoplasia & - & 1 & - & - & 1 & - & - & 1 & 1 & - & 1 & - & 5 \\
\hline Doenças hematológicas & 1 & 1 & - & 2 & - & - & 2 & 1 & 2 & 3 & 1 & 3 & 16 \\
\hline Doenças endócrinas & 2 & 1 & 1 & - & 1 & - & 1 & 3 & 2 & - & 1 & 2 & 14 \\
\hline Doenças do aparelho respiratório & 3 & 5 & - & - & 4 & 3 & 2 & 2 & 2 & 4 & 3 & 4 & 32 \\
\hline Doenças do aparelho digestório & 1 & 1 & 1 & 1 & - & - & 1 & 1 & - & - & - & - & 6 \\
\hline Doenças do aparelho geniturinário & - & - & - & - & - & - & 1 & - & - & - & - & - & 1 \\
\hline Doenças do sistema nervoso & 1 & - & - & 1 & 3 & 1 & 2 & 2 & 2 & - & 1 & 1 & 14 \\
\hline $\begin{array}{l}\text { Problemas decorrentes de } \\
\text { malformação congênita }\end{array}$ & - & 1 & 2 & 1 & 2 & 2 & 3 & 3 & 4 & 1 & 3 & 1 & 23 \\
\hline $\begin{array}{l}\text { Doenças não discriminadas originadas } \\
\text { no perinatal }\end{array}$ & 11 & 6 & 11 & 16 & 11 & 11 & 1 & 2 & - & - & 3 & 2 & 74 \\
\hline $\begin{array}{l}\text { Diarréias, gastroenterites de infecções } \\
\text { presumíveis }\end{array}$ & - & 1 & - & - & 1 & - & - & 2 & 3 & 1 & - & 2 & 10 \\
\hline Total & 28 & 23 & 18 & 21 & 26 & 18 & 15 & 20 & 20 & 12 & 16 & 18 & 235 \\
\hline
\end{tabular}

acerca da indicação dessa vacina para imigrantes de áreas indenes Que pretendem visitar ou instalar-se em áreas de risco.

Em relação às demais vacinas, a cobertura sofreu peQuenas oscilações em relação a meta vacinal de pelo menos 95\%. Dados Que são reforçados pela Secretaria de Saúde da Paraíba ${ }^{(8)}$ Quando afirma Que até outubro de 2003 havia baixa cobertura vacinal, exceto para BCG e tríplice viral Que alcançaram a meta 100\%. E Que aconteceu Queda no alcance das coberturas vacinais da DTP + Hib e Hepatite B em toda a Paraíba entre 2003 e 2004, tal como demonstrada no Quadro 2. A explicação estatal para a inconsistência dos dados é a de que a base populacional utilizada na estimativa do IBGE 2004 encontra-se superestimada em muitos municípios, impossibilitando o alcance da meta pactuada. Esse fenômeno motivou a indicação da revisão de erros de registros de doses aplicadas nas informações apresentadas ao Estado.

Em se tratando da morbi-mortalidade infantil, esse agravo costuma ser conseQüência do impacto do meio ambiente sobre a criança causando infecções respiratórias agudas, diarréia e as doenças imunopreveníveis. O primeiro ano de vida é aquele de maior risco de morte devido a vulnerabilidade às condições de vida e de acesso a bens e serviços, razão porque o CMI tem sido considerada um indicador sensível às condições sociais e de saúde das populações humanas. No caso específico de Campina GrandePB, as causas de óbito infantil estão apresentados na Tabela 3.

De acordo com estes dados, verifica-se Que ao longo do período estudado, ocorreu a redução da mortalidade infantil em relação às doenças infecciosas ou parasitárias e às doenças não discriminadas originadas no perinatal. Os óbitos pelas demais patologias registradas mantiveram uma tendência a prevalência dos óbitos.

Nesse estudo não foi possível identificar se os óbitos por doenças infecciosas decorreram de doenças imunopreveníveis, pois as informações obtidas não faziam referência a causa mortis. Também não foi possível identificar Quais as patologias Que determinaram o óbito infantil no perinatal. Entretanto, detectou-se em relato da literatura Que as doenças do aparelho respiratório merecem destąue como as principais causas de internações hospitalares: a infecção respiratória aguda (IRA) atinge o percentual 27,7\%; outros tipos de infecção respiratória, Quando somados, alcançam o percentual 13,8\%. lá as doenças infecciosas e parasitárias são da ordem de $16,9 \%{ }^{(9)}$

A situação de saúde das pessoas mantém estreita relação com o estilo de vida, as condições socioeconômicas e, portanto, com os espaços onde se manifesta a articulação entre os processos biológicos e sociais Que determinarão o processo saúde-doença 
em uma sociedade dada ${ }^{(10)}$.

O declínio da mortalidade nos países em desenvolvimento passa pelas ações explicitas de saúde pública, especialmente, pelo combate a doenças infecciosas e endêmicas. Medidas de saúde pública, campanha de imunização, uso de antibióticos, pulverização com inseticida para erradicar as doenças causadas por insetos transmissores, a clarificação da água, sanificação do meio, suplementação alimentar, entre outros. Dessa forma, observa-se uma tendência a redução da mortalidade infantil devido ao implemento de um sistema de saúde que se propõe a assegurar aos cidadãos equidade, universalidade e igualdade no acesso. E do desenvolvimento de políticas estatais voltadas para a redução das desigualdades sociais.

Em relação a mortalidade por causas perinatais, essa ainda representa o principal contingente das mortes no primeiro ano de vida. Os fatores determinantes desse tipo de óbito encontram-se intimamente associados às condições de saúde da gestante e à Qualidade dos cuidados prestados durante o pré-natal, o parto e a assistência ao recém-nascido( ${ }^{(7)}$.

A preocupação da Área Técnica de Saúde da Criança e Aleitamento Materno do Ministério da Saúde com os fatores determinantes do óbito perinatal motivou a criação, em junho de 2006, da Rede Norte Nordeste de Saúde Perinatal

Que conta com o apoio do Núcleo de Ensino, Assistência e Pesquisa da Infância César Victora (NEAPI) e da Disciplina de Pediatria do Departamento Materno-Infantil da Faculdade de Medicina da Universidade Federal do Ceará. Esses parceiros objetivam promover a melhoria do desempenho das Unidades Neonatais de Médio e Alto Risco e reduzir a morbi-mortalidade evitável na região Norte-Nordeste do país.

Por enquanto, essa Rede está implementada nos 9 estados do Nordeste, ficando os estados do Norte para o ano de $2007^{(7)}$.

Ainda em relação a análise do Quadro 3, a subnotificação de dados referentes aos óbitos por doenças infecciosas e perinatais causou prejuízo na produção de dados diretos fidedignos acerca do conhecimento preciso das doenças Que acometeram as crianças, confirmando a opinião de autores acerca de que, na maioria dos estados brasileiros, o uso dos sistemas de informação é passível de limitação ${ }^{(1)}$.

Para além dessa lacuna, utilizou-se os dados da Tabela 3 para o cálculo do CMI em Campina Grande-PB de modo Que, ao longo do período estudado, partindo-se do ano 2000 até 2005, o coeficiente de mortalidade infantil apresentou a seguinte seQüência: 15,58; 11,51; 13,01; 10,87; 10,49; e 10,97.

Relato da literatura considera ${ }^{(12)}$ Que o CMI é alto Quando ele alcança 50 óbitos para cada mil nascidos vivo; é médio Quando oscila entre 20 a 49 e baixo Quando for menor que 20. Quando o CMI é alto a mortalidade predomina no período pós-neonatal e reflete baixos níveis de saúde e desenvolvimento socioeconômico. Quando o CMI é baixo a mortalidade prevalece no período neonatal precoce e pode ter relação com más condições de vida em segmentos sociais específicos. Assim, considera-se Que esse coeficiente é um forte indicador social, pois, Quanto piores as condições de vida, maior a taxa de mortalidade e menor a esperança de vida.

De acordo com o cruzamento dos CMI detectados nesse estudo com aQueles da classificação desse tipo de coeficiente detectada na literatura, confirma-se Que a cobertura vacinal das crianças menores de um ano de vida em Campina Grande-PB causou impacto, de modo Que, nos últimos anos o município vem apresentando baixo coeficiente de mortalidade infantil.

Além da cobertura vacinal, a redução da mortalidade infantil em Campina Grande-PB também está relacionada com a melhoria das condições do meio ambiente, da Qualidade de vida da população e com o implemento da assistência em saúde propiciada pela ampliação da rede básica de saúde.

O impacto da cobertura vacinal em Campina Grande condiz com o compromisso brasileiro com os Objetivos do Milênio pactuado pelos 191 Estados-Membros das Nações Unidas Que, dentre outras metas, pretende reduzir em 2/3 a mortalidade de menores de 5 anos até o ano de 2015. E com o Pacto pela saúde de 2006 Que, também, prioriza as ações destinadas a reduzir a mortalidade infantil(7)

\section{Recursos disponíveis para o planejamento das ações de imunização infantil}

Os enfermeiros atuantes nas 62 UBSF visitadas contam com recursos humanos $(47,19 \%)$ das respostas; cadastro familiar (13,48\%); material de divulgação de atividades (2 1 ,35\%); insumos e imunizantes (12,36\%); e disponibilidade de sala de vacina (5,62\%).

A carência de recursos no campo da imunização exige da equipe da atenção básica a articulação com os diversos setores envolvidos na promoção da saúde visando a elaboração e a adoção daQuelas medidas Que se fizerem necessárias para melhora o desempenho das atividades sanitárias e propiciar atenção condizente com as reais necessidades da população. Isto porQue Quando os profissionais de saúde trabalham em condições precárias de assistência esse fato prejudica o desempenho das equipes, afeta a Qualidade dos serviços prestados e o grau de satisfação dos usuários. E se contrapõe a política de humanização ${ }^{(13)}$ Que preconiza a valorização dos diferentes sujeitos comprometidos com a produção de saúde.

Outrossim, as equipes se Queixaram da falta nas UBSF de instrumentos para distrair as crianças na hora da vacinação (87\%). Essa lacuna é preocupante porQue em relato da literatura detectouse Que antes e durante a vacinação intramuscular as crianças apresentaram as reações: procura de ajuda, reação de pânico, expressão verbal e motora de medo, movimento da musculatura facial, choro prolongado, explosão de grito, rigidez muscular ${ }^{(14)}$.

\section{Barreiras enfrentadas pelos enfermeiros para alcançar a meta $100 \%$ de vacinação infantil proposta pelo PNI}

As dificuldades encontradas pelos vacinadores para atingir a cobertura vacinal estão apresentadas na Tabela 4 .

A resistência dos pais foi apontada como a principal barreira para o alcance da cobertura vacinal podendo esta ser fruto das crenças, cultura dos mais velhos e até mesmo o medo da reação da vacina. Além da resistência paterna, foram citadas: falta de ACS, pois, em áreas descobertas pelo ACS não é possível se saber o real número de crianças menores de um ano; e a falta material. Esta última foi apontada como uma das causas para Que as mães não levem seus filhos com freeüência à unidade básica de saúde para imunização.

As barreiras ao alcance da cobertura vacinal detectadas nesse estudo assemelham-se àQuelas detectadas por outros autores: falta de transporte, de infra-estrutura, de equipamentos, de médicos especialistas, de medicamentos, de apoio dos gestores, de vontade 
Tabela 4. Dificuldades enfrentadas pelos enfermeiros para atingir a meta vacinal.

\begin{tabular}{lc}
\hline Dificuldades & $\%$ \\
\hline Falta ACS & $22 \%$ \\
Falta insumos & $22 \%$ \\
Falta sala de vacina & $7 \%$ \\
Falta imunobiológicos & $13 \%$ \\
Resistência dos pais à vacinação & $26 \%$ \\
Desconhecimento da meta & $2 \%$ \\
Falta mobilização dos profissionais & $4 \%$ \\
Dificuldades de acesso & $2 \%$ \\
Não há dificuldades & $2 \%$ \\
\hline
\end{tabular}

política, e de mais profissionais ${ }^{(15)}$.

\section{Ações desenvolvidas pela equipe das UBS para reduzir a morbi- mortalidade infantil}

A conduta dos profissionais atuantes nas UBS estudadas para reduzir a morbi-mortalidade infantil, envolve ações preconizadas tanto pela estratégia Atenção Integrada às Doenças Prevalentes na Infância(AIDPI) ${ }^{(15)}$ como pela Agenda de Compromissos para a Saúde Integral da Criança e Redução da Mortalidade Infantil (ACSICRMI) $^{(16)}$. No caso específico das ações preconizadas pelo Programa Nacional de Imunizações(PNI) destacam-se: planejamento e execução das ações de vacinação e controle da rede de frio, integração da vacinação às ações básicas de saúde, acompanhamento e avaliação das metas de cobertura vacinal e a garantia da homogeneidade das coberturas vacinais na região de atuação profissional ${ }^{(7)}$.

Os percentuais relativos às ações desenvolvidas nas UBS desse estudo são da seguinte ordem: busca ativa das crianças com vacinação atrasada $(4,14 \%)$; ações de puericultura $(30,34 \%)$; incentivo ao aleitamento materno (1 1,03\%); educação em saúde $(13,10 \%)$; vacinação infantil $(13,79 \%)$; vigilância a doenças da infância $(4,82 \%)$; visita domiciliar $(8,97 \%)$; assistência a grupo de gestantes (2,07\%); assistência pré-natal $(9,66 \%)$; atua em programa de vit. A e ferro $(4,14 \%)$.

À luz da opinião de autores ${ }^{(17)}$ acerca de Que a atenção básica às crianças menores de um ano de idade exige promoção da prática de amamentação, redução e controle da morbimortalidade infantil, alcance da cobertura vacinal, estímulo do apego-mãe-filho-família, inserção da família nos cuidados, além de organização dos serviços e capacitação dos recursos humanos como reQuisitos para o avanço na assistência integral à saúde da criança e da família, os autores desse estudo acreditam Que há necessidade de maior articulação entre os atores da gestão municipal, estadual, e federal no sentido de melhorar o desempenho profissional nas ações desenvolvidas nas UBS alvo desse estudo.

\section{CONCLUSÃO}

A vacinação infantil no primeiro ano de vida é fundamental para a prevenção de várias doenças transmissíveis e é um dos fatores determinantes da redução do Coeficiente de Mortalidade Infantil. A identificação da cobertura vacinal e dos fatores responsáveis pelo retardo ou pela falta de imunizações é ação fundamental para a adęuada monitorização dos programas de vacinação e para se identificar e atingir as crianças Que não são vacinadas.

A pesquisa apontou Que no espaço temporal selecionado para o estudo há dispersão em relação ao número de crianças cadastradas nas UBS de Campina Grande-PB e o número de nascidos vivos nesse mesmo período. Que o município extrapolou a cobertura vacinal com a BCG e a anti-pólio. Mas, em relação às demais vacinas ocorreram oscilações percentuais durante todo o período, ora aproximando-se da meta, ora afastando-se.

O controle da morbi-mortalidade infantil é realizado com o incremento dos recursos humanos disponíveis para o planejamento das ações a serem implementadas. E ocorre déficit de recursos materiais. As ações desenvolvidas objetivando esse controle condizem com aQuelas preconizadas pelo AIDPI e pela Agenda de Compromissos para a Saúde Integral da Criança e Redução da Mortalidade Infantil.

A mortalidade infantil decresceu, de modo Que o cálculo da média do O CMI alcançou índices abaixo de 20, portanto, classificado como baixo ICM, confirmando o impacto da cobertura vacinal em crianças menores de um ano de vida em Campina GrandePB. A resistência dos pais a vacinação, a falta de insumos como seringas e agulhas,e de ACS em algumas UBS, foram as principais barreiras enfrentadas pelas equipes das UBSF para atingir a meta vacinal proposta pelo PNI.

Ante o exposto, entende-se Que a ampliação do número de ACS para as UBSF seria de suma importância, tendo em vista que esses profissionais possuem um poder maior de persuasão e mobilização social, tendo em vista Que os mesmos pertencem às mesmas comunidades em Que atuam. Que as equipes precisam desenvolver estratégias que visem o esclarecimento da comunidade no que diz respeito aos benefícios advindos da vacinação, tendo em vista Que a rejeição da família pode ser decorrente da falta de esclarecimento das pessoas sobre o real intuito da vacinação.

Entende-se, ainda, Que o cumprimento do calendário de vacinação, pré-requisito para Que a população seja contemplada com programas sociais do Governo, a exemplo da Bolsa Família, sem dúvida, tem feito com Que os pais se preocupem mais com esta Questão. No entanto, partindo-se do pressuposto de Que a prevenção é uma das formas mais eficazes e econômicas de cuidar da saúde, é conveniente que o Governo Federal e, por conseqüência, o Estadual e o Municipal, busquem novas formas de benefícios, Que possam atrair cada vez mais a população de baixa renda.

\section{REFERÊNCIAS}

1. Ministério da Saúde (BR). Programa Nacional de Imunizações: 30 anos. Brasília: Ministério da Saúde; 2003.

2. Ministério da Saúde (BR). Secretaria de Vigilância em Saúde. Sistema de Informações sobre Nascidos Vivos. Indicadores de mortalidade. IDB 2006 Brasil Taxa de mortalidade infantil.

Brasília: Ministério da Saúde; 1996.

3. Brasil. Portaria № 597, de 8 de abril de 2004. Institui, em todo o território nacional, os calendários de vacinação. Diário Oficial da República Federativa do Brasil 2004 abr; 69(1): 46.

4. Ministério da Saúde (BR). Estatuto da Criança e do Adolescente. 
3a ed. Brasília: Ministério da Saúde; 2007.

5. Instituto Brasileiro de Geografia e Estatística. Censo demográfico 2000. Rio de Janeiro: IBGE; 2000.

6. Secretaria de Saúde de Campina Grande (PB). Sistema de Informação de Atenção Básica. Campina Grande: Coordenação de Imunização; 2005.

7. Ministério da saúde (BR). Redução da Mortalidade Infantil. Brasília: Ministério da Saúde; 2005.

8. Ministério da Saúde(BR). Secretaria de Vigilância Sanitária em saúde. Sistema Nacional de Vigilância em saúde: relatório de situação: Paraíba. Brasília: Ministério da Saúde; 2005.

9. Caetano IRM, Bordin IAS, Puccini RF, Peres CA. Fatores associados à internação hospitalar de crianças menores de cinco anos. Rev Saúde Pública 2002; 36(3): 285-91.

10. Araújo MRN, Assunção RS. A atuação do Agente Comunitário de Saúde na promoção da saúde e na prevenção de doenças. Rev Bras Enferm 2004; 57(1): 19-25.

11. Ministério da Saúde (BR): Rede Interagencial de Informações para a Saúde. Indicadores básicos para a saúde no Brasil: conceitos e aplicações. Brasília: Ministério da Saúde; 2007.
12. Pereira MG. Epidemiologia: teoria e prática. Rio de Janeiro: Guanabara Koogan; 1995.

13. Ministério da Saúde (BR). Secretaria de Atenção à Saúde. Núcleo Técnico de Política Nacional de Humanização. Trabalho e redes de saúde: valorização dos trabalhadores da saúde. $2^{\mathrm{a}}$ ed. Brasília: Ministério da Saúde; 2006.

14. Santos MCN, Borba RIH, Sabatés AL. A importância do preparo da criança pré-escolar para a injeção intramuscular com o uso do brinquedo. Acta Paul Enferm 2000; 13(2): 52-8.

15. Ministério da Saúde (BR). AIDPI Atenção Integrada às Doenças Prevalentes na Infância: curso de capacitação: introdução ao módulo I. Brasília: Ministério da Saúde; 2004.

16. Ministério da Saúde (BR). Secretaria de Atenção à Saúde. Departamento de Ações Programáticas Estratégicas. Agenda de Compromissos para a Saúde Integral da Criança e Redução da Mortalidade Infantil. Ministério da Saúde. Secretaria de Atenção à Saúde. Departamento de Ações Programáticas Estratégicas. Brasília: Ministério da Saúde; 2005.

17. Lima VM, Mello DF. Assistência de enfermagem a crianças menores de um ano de idade em unidade básica de saúde. Rev Bras Enferm 2004; 57(5): 531-3. 